\title{
The use of rank adjustment procedure to constrain climate projections from a given multi model ensemble
}

\author{
Shih-Chung Tai and Yung-An Lee*
}

Department of Atmospheric Sciences, National Central University, Taoyuan City, Taiwan

\begin{abstract}
Article history:
Received 16 June 2020

Revised 20 August 2020

Accepted 21 August 2020

Keywords:

Climate projection, Uncertainty range, Emergent constraint, Rank adjustment

Citation:

Tai, S.-C. and Y.-A. Lee, 2020: The use of rank adjustment procedure to constrain climate projections from a given multi model ensemble. Terr. Atmos. Ocean. Sci., 31, 649-662, doi: 10.3319/TAO.2020.08.21.01
\end{abstract}

\begin{abstract}
In this study, we develop a three steps rank adjustment procedure to constrain future projections of interest. This procedure uses nonparametric present-future relations from a given multi model ensemble (MME) to estimate the rank distribution of observations in future projection period to constrain the corresponding projections. We then applied this rank adjustment procedure to constrain future projections of the global mean surface temperature (GMST) as well as the surface temperature and the precipitation fields from the CMIP5 Representative Concentration Pathway 8.5 (RCP85) scenario MME. For the GMST, we successfully narrow the 5 - 95 uncertainty range by one-half at the end of the 21 st century. For the surface temperature field, the constrained MME medians averaged over 2081 - 2100 exhibit more smooth and homogeneous spatial variations than those original MME medians. More interestingly, the corresponding projected precipitation field are completely free from the long-standing double ITCZ bias. These results suggest that the use of rank adjustment procedure is capable of yielding more consistent future projections for both the surface temperature and the precipitation fields. More importantly, because the rank adjustment procedure is based on nonparametric present-future relations of a given MME, one expects that it have wider range of applicability than previously identified and proposed emergent constraints.
\end{abstract}

\section{INTRODUCTION}

Climate is a one of the major factors affecting human well-being and economic productivities around the world (Carleton and Hsiang 2016). To mitigate and adopt climate change induced social and economic impacts, we need to estimate the magnitude and associated uncertainty range of future climate change under various radiative forcing scenarios. For this purpose, simulation results from multi model ensembles (MMEs) of the Coupled Model Intercomparison Project (CMIP) are valuable data sources. Many efforts have been made to integrate MME results into combined projections that represent some consensus view with associated uncertainty during different phases of the CMIP (Tebaldi and Knutti 2007; Knutti 2008, 2010; Sanderson et al. 2015). The mostly common approach for dealing with MME results is model democracy (i.e., "one model one vote") (Knutti 2010). Thus, the 'best-guess' and uncertainty ranges of fu-

\footnotetext{
* Corresponding author

E-mail:yalee@atm.ncu.edu.tw
}

ture projection of a given climate variable are estimated using the simple ensemble mean and the 5 - 95 range of model spread from MME of the CMIP5 (Taylor et al. 2012). However, CMIP5 models are not independent (Knutti et al. 2013; Masson and Knutti 2011). Furthermore, research organizations simply submit as many simulations as they are able to. Therefore, it is extremely unlikely that the corresponding multi model mean is in any way optimal (Herger et al. 2018). Although Bayesian methodologies (Robertson et al. 2004) and model weighting (Krishnamurti et al. 2000) have been used for model combination, but the correct implementation and interpretation of such studies is subject to some debate (Tebaldi and Knutti 2007; Sanderson and Knutti 2012).

Recently, the emergent constraint approach that offers hope to constrain future projections of quantities of interest is gaining popularity in climate models evaluation (Hall and Qu 2006; Bracegirdle and Stephenson 2012; Caldwell et al. 2014; Hall et al. 2019). Briefly speaking, emergent constraints are empirical relationships between current climate and future prediction emerging from collections of 
climate model simulations that are physically explainable (Klein and Hall 2015). Thus, by selecting a set of models that are consistent with observations, these empirical relationships can be used to constrain the range of possible future projections (Borodina et al. 2017). However, some studies (Abe et al. 2009; Knutti et al. 2010; Caldwell et al. 2014) showed that the correlation between the present-day and future climate patterns exhibited in models generally was not significant. Furthermore, the lack of independence of CMIP ensemble members may create artificial correlations and the screening of predictors will likely find correlations that have no physical basis (DelSole and Shukla 2009; Masson and Knutti 2013; Caldwell et al. 2014; Sanderson et al. 2015). Moreover, most emergent constraints have been proposed or identified so far are linear (Hall et al. 2019). Because the earth climate is a highly nonlinear system, it is likely that a substantial fraction of relations between future changes and current states may also be nonlinear. Therefore, we may need to find methods other than conventional regression method to cope with such situations.

The rank histogram (Anderson 1996; Hamill and Colucci 1997; Hamill 2001) is a widely used technique for evaluating the reliability or calibration of an ensemble forecast. A rank histogram is constructed by using the rank statistics of the observation in an ensemble (Anderson 1996). Because ranking is a nonparametric concept, this characteristic renders the rank histogram a flexible tool to be used for assessing the calibration (Mirzargar and Anderson 2017). This nonparametric characteristic also leads us to wonder whether some kinds of relationships between rank distributions of a given MME in historical and future projection periods can be established. If such relations do exist, then we may be able to use them to constrain the corresponding future projections with current observations. Therefore, one of the main purpose of this study is to explore the feasibility of using the rank distributions of a given MME to establish nonparametric present-future relations to be used in constraining the corresponding future projections. Furthermore, because adjustments using emergent constraints can remove the effect of historical simulation errors on future climate projection in each model (Li et al. 2016), the use of anomalous time series with/without mean bias removed may have significant impact on calibrated results. Hence, the other purpose of this study is to examine the effect of different data preprocessing procedures on constrained results.

Because the surface temperature and the precipitation are the two variables that have the most social-economic influences, in this study we examine if the proposed nonparametric present-future relation of a MME can be used to constrain the corresponding future projections of these two variables. The remainder of this paper is organized as follows. We first describe data and the two data preprocessing procedures used in this study in section two. Then, we introduce the methodology and implementation of the three steps rank adjustment procedure in section three. In section four, we present and compare the original and the rank adjustment constrained results of the global mean surface temperature (GMST) anomalies, the surface temperature and the precipitation fields. Finally, we summarize and conclude this study in section five.

\section{DATA}

Observed data used in this study are monthly mean surface temperature anomalies in the period $1901-2018$ from the Met office Hadley Centre HadCRUT4.6 (Morice et al. 2012) and monthly mean precipitation in the period 1979 - 2018 from the CPC Merged Analysis of Precipitation (CMAP) (Xie and Arkin 1997). Model data are monthly mean surface temperature (tas) and monthly mean precipitation (pr) in the period $1901-2100$ from all simulations of the Representative Concentration Pathway 8.5 scenario in the CMIP5 dataset of the KNMI Climate Explorer website (https://climexp.knmi.nl/start.cgi) and are denoted as the RCP85 MME. Table 1 lists the number of simulations of each variable for each CMIP5 model. In all, there are 81 and 77 members in the RCP85 MME for surface temperature and precipitation, respectively. Furthermore, all original observed and model data were regridded to a common resolution of $5^{\circ} \times 5^{\circ}$ grid prior to further analyses.

To examine the effect of different data preprocessing procedures on constraining future projections, two anomalous datasets were generated from the original RCP85 MME in this study. The first dataset is generated by removing a common reference state from each original time series of the RCP85 MME and is denoted as the RCP85-clim MME. The common reference state for the surface temperature field is the same as the climatological reference state of the HadCRUT4.6 (i.e., the 1961 - 1990 mean seasonal cycle). However, because the CMAP data are only available from 1979 onward, the same 1961 - 1990 period cannot be used as the reference period, therefore we choose the 1981 - 2010 mean seasonal cycle of the CMAP as the common reference state for the precipitation field. The second dataset is generated by removing each individual time series' own mean seasonal cycle from the original RCP85 MME and is denoted as the RCP85-individual MME. The mean seasonal cycle of each time series is defined in the same corresponding reference period as above.

To demonstrate the distinct effects of these two data preprocessing on temporal behavior of the original MME, Fig. 1 shows temporal evolutions of the GMST from the original RCP85 MME as well as the GMST anomalies (gmsta) from the RCP85-clim MME and the RCP85-individual MME, respectively. It is noted that because the only difference between the GMST in Fig. 1a and the gmsta in Fig. 1b is the common mean seasonal cycle, therefore, time series in both panels exhibit similar temporal evolutions and 
warming trends. Furthermore, the spreads of these two MMEs are over-dispersive compared to temporal variability of the HadCRUT4.6 but remains approximately constant over time and the corresponding MME means exhibit slightly cold biases compared to the observations. Hence this kind of data preprocessing in generating the gmsta of the RCP85-clim MME will preserve almost all temporal characteristics of the original data including the mean bias of each original time series. On the other hand, because the gmsta in Fig. 1c were generated by removing each individual time series' own mean seasonal cycle in period 1961 - 1990 from the GMST of the original RCP85 MME, the mean bias of each original time series in that reference period is also removed. Thus, although clear warming trends are still observed, those anomalies in Fig. 1c tend to concentrate around the observations in period 1961 - 1990 but gradually disperse toward both ends. Furthermore, all anomalous time series in Fig. 1c exhibit no visible mean biases in historical period. These results suggest that this kind of data preprocessing in generating the gmsta of the RCP85-individual MME can remove the mean bias of the reference period from each original time series but cannot preserve the relative positions (ranking) of each time series in the original RCP85 MME.

\section{METHODOLOGY}

In this study, we develop a three steps rank adjustment procedure to explore the feasibility of using nonparametric present-future relationships of a given MME to constrain the corresponding future projections. The first step is to establish nonparametric present-future relations from the rank distributions of future projection and historical periods for all members of a given MME. The second step is to construct the rank distribution of the observations in the same historical period to be used to constrain the corresponding future projections. The third step is to adjust the rank distribution of the observations in the future projection period using the established present-future relationships to constrain the 'best-guess' and uncertainty ranges of future projections from the corresponding MME. A brief description of detailed implementation for each step is as follows.

\subsection{Establish Nonparametric Present-Future Relationships}

To establish a present-future relationship of a given variable at a specific grid, we first apply the perfect model approach (Abramowitz and Bishop 2015; Knutti et al. 2017) to calculate the rank difference between future projection period (2051 - 2100) and historical period (1951 - 2000) for all members of either the RCP85-clim MME or the RCP85individual MME. For a given MME, the basic concept of the perfect model approach is to treat each member in turn as 'truth' and the rest of the MME as the corresponding
Table 1. The number of RCP8.5 simulations from each CMIP5 model.

\begin{tabular}{|c|c|c|c|}
\hline Model number & Model Name & RCP8.5 tas & RCP8.5 pr \\
\hline 1 & ACCESS1-0 & 1 & 1 \\
\hline 2 & ACCESS1-3 & 1 & 1 \\
\hline 3 & BCC-CSM1-1 & 1 & 1 \\
\hline 4 & BNU-ESM & 1 & 1 \\
\hline 5 & CanESM2 & 5 & 5 \\
\hline 6 & CCSM4 & 6 & 6 \\
\hline 7 & CESM1-BGC & 1 & 1 \\
\hline 8 & CESM1-CAM5 & 2 & 2 \\
\hline 9 & CMCC-CM & 1 & 1 \\
\hline 10 & CMCC-CMS & 1 & 1 \\
\hline 11 & CNRM-CM5 & 5 & 5 \\
\hline 12 & CSIRO-Mk3.6.0 & 10 & 10 \\
\hline 13 & EC-EARTH & 8 & 4 \\
\hline 14 & FGOALS-g2 & 1 & 1 \\
\hline 15 & FIO-ESM & 3 & 3 \\
\hline 16 & GFDL-CM3 & 1 & 1 \\
\hline 17 & GFDL-ESM2G & 1 & 1 \\
\hline 18 & GFDL-ESM2M & 1 & 1 \\
\hline 19 & GISS-E2-H p1 & 1 & 1 \\
\hline 20 & GISS-E2-H p2 & 1 & 1 \\
\hline 21 & GISS-E2-H p3 & 1 & 1 \\
\hline 22 & GISS-E2-R p1 & 1 & 1 \\
\hline 23 & GISS-E2-R p2 & 1 & 1 \\
\hline 24 & GISS-E2-R p3 & 1 & 1 \\
\hline 25 & HadGEM2-AO & 1 & 1 \\
\hline 26 & HadGEM2-CC & 1 & 1 \\
\hline 27 & HadGEM2-ES & 4 & 4 \\
\hline 28 & INMCM4 & 1 & 1 \\
\hline 29 & IPSL-CM5A-LR & 4 & 4 \\
\hline 30 & IPSL-CM5A-MR & 1 & 1 \\
\hline 31 & IPSL-CM5B-LR & 1 & 1 \\
\hline 32 & MIROC5 & 3 & 3 \\
\hline 33 & MIROC-ESM & 1 & 1 \\
\hline 34 & MIROC-ESM-CHEM & 1 & 1 \\
\hline 35 & MPI-ESM-LR & 3 & 3 \\
\hline 36 & MPI-ESM-MR & 1 & 1 \\
\hline 37 & MRI-CGCM3 & 1 & 1 \\
\hline 38 & NorESM1-M & 1 & 1 \\
\hline 39 & NorESM1-ME & 1 & 1 \\
\hline
\end{tabular}



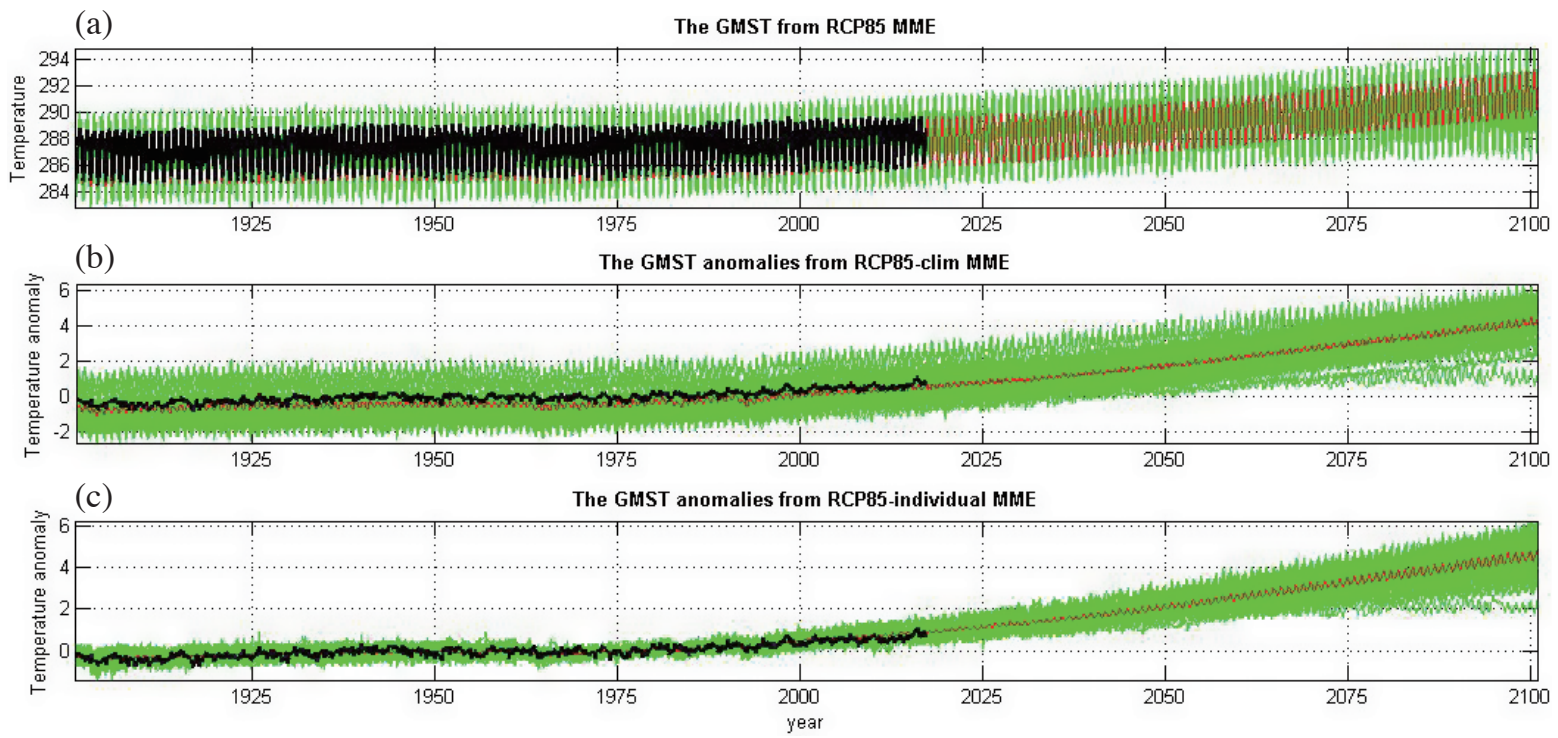

Fig. 1. Temporal evolutions of the GMST from the original RCP85 MME (a) as well as the gmsta from the RCP85-clim MME (b) and the RCP85individual MME (c), respectively. In each panel, the green lines are all time series from the given MME, while the thick black and red lines are the corresponding time series from the HadCRUT4.6 and the given MME mean, respectively.

MME. Then, we can determine the rank distribution of each 'truth' within the given MME in any given period. Take the gmsta of the RCP85-clim MME in historical period (1951 $2000)$ as an example. Because there are 81 members in the MME and each member has 600 time steps, this example dataset forms a $81 \times 600$ matrix A. By sorting each column of $\mathbf{A}$ in ascending order and identifying the corresponding column position of the 'truth', we yield a time series consisting 600 ranks of the 'truth'. From this time series we can then construct the rank histogram of the 'truth' in historical period. Therefore, by treating each member in turn as 'truth' and applying the same data manipulations as above, we can obtain the rank distributions for all members of the given MME at both historical and future projection periods. Afterward, we calculate rank differences of each member between future projection and historical periods at 5 th, 10 th, 20th, 30th, 40th, 50th, 60th, 70th, 80th, 90th, and 95th percentiles. Taking the 5th percentile of the RCP85-clim MME as an example, we first calculate the rank of 5th percentile from 600 ranks of a given model in period 1951 - 2000 (rank A). Similarly, we calculate the rank of 5th percentile from 600 ranks of a given model in period $2051-2100$ (rank B). Then the corresponding rank difference is defined as rank B-rank A. The same calculations are repeated for every member of the given MME.

As a demonstration, Fig. 2 shows boxplots of rank differences between future projection and historical periods as a function of percentiles for the original RCP85 GMST, the RCP85-clim gmsta, and the RCP85-individual gmsta. One clearly observes that boxplots in Figs. 2a and b are identical. This is expected because the data preprocessing in deriving the RCP85-clim gmsta does not alter the rank distribution of the original RCP85 MME. More interestingly, one notes that all medians of rank differences in these two panels are very close to zero. It is noted that rank differences between future projection and historical periods can be viewed as the corresponding nonparametric trends of each member at these percentiles. This implies that the medians of rank differences of all members of a given MME can be viewed as the optimal estimation of the nonparametric trends of the given MME at these percentiles. Therefore, median of rank differences from each examined percentile is the desired nonparametric present-future relation we want to establish. From this perspective, all medians of rank differences in both Figs. 2a and b imply that both the original RCP85 MME and the RCP85-clim MME exhibit no preferred nonparametric trends at all examined percentiles and are in agreement with the almost constancy of MME spreads observed in Figs. 1a and b. In contrast, almost all medians of rank differences in Fig. 2c are significantly different from zero. This result indicates that the RCP85-individual MME has notable nonparametric trends at all examined percentiles. Thus, the rank distribution of this MME is apparently inconsistent with that of the original MME. Because the mean bias of each member of the original RCP85 MME in period 1961 - 1990 were removed, this result implies that this kind of data preprocessing renders the rank distribution of the RCP85-individual MME being significantly deviated from that of the original RCP85 MME.

Base on the above results, it appears that the closeness to zero of most medians of rank differences from all examined percentiles can be used to identify whether the 

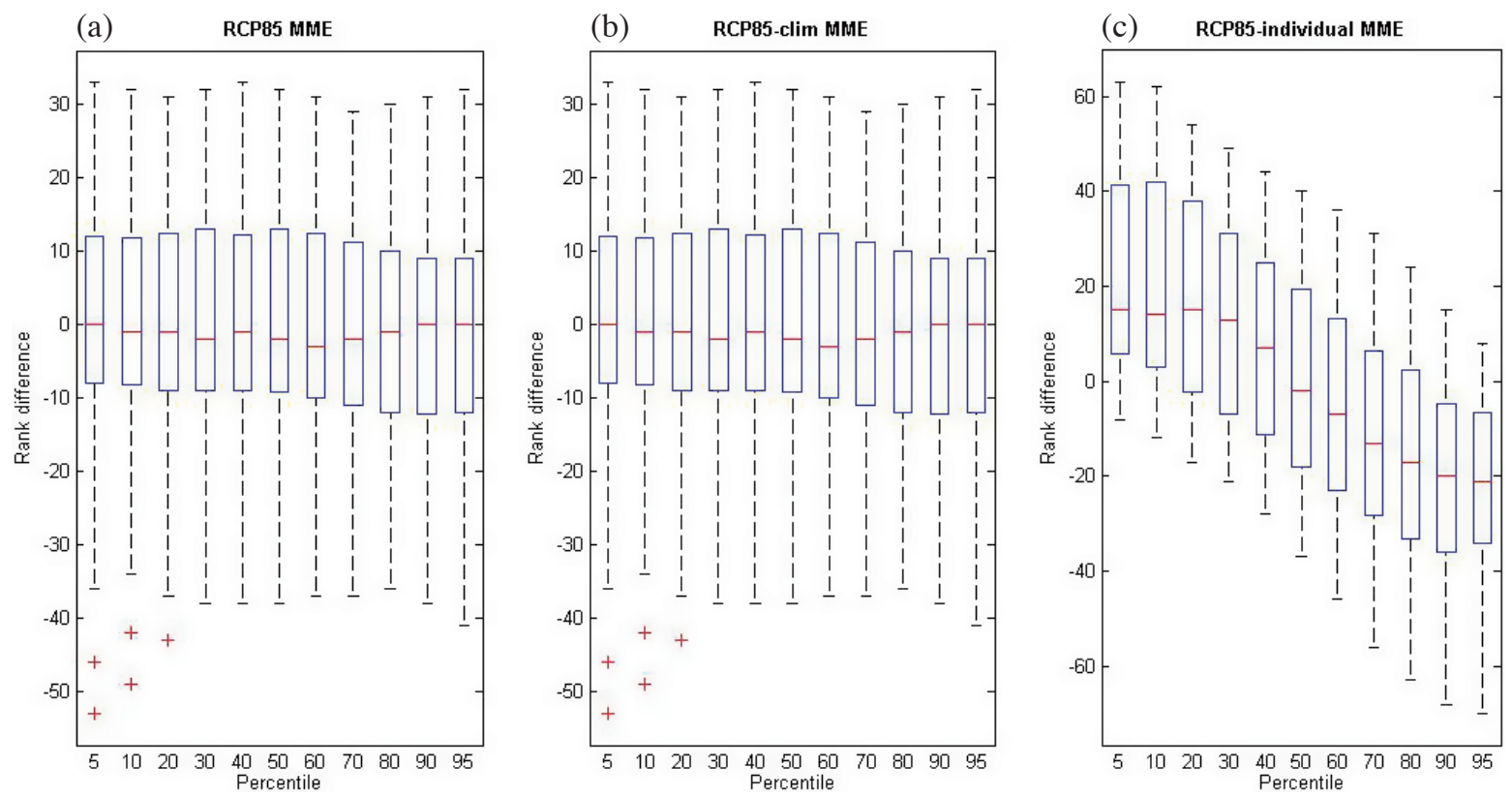

Fig. 2. Boxplots of rank differences between future projection period (2051 - 2100) and historical period (1951 - 2000) as a function of percentiles from the original RCP85 MME (a), the RCP85-clim MME (b), and the RCP85-individual MME (c), respectively. Each box and the red line within the box correspond to the interquartile range (IQR) and the median value at the given percentile. Because there are 81 members in each MME, therefore each boxplot consists of 81 samples.

rank distribution of a given MME is consistent with the constancy of rank distribution condition. Therefore, in this study we define a rank constancy indicator $(\bar{\gamma})$ as the average of absolute values of the medians of rank differences from all examined percentiles and the closeness to zero of $\bar{\gamma}$ may be used to identify whether the rank distribution of a given MME is consistent with the constancy condition. The formula of $\bar{\gamma}$ is as follows. Let $\gamma_{i}$ be the median of rank difference of the $i$-th examined percentile and $m$ is the total number of examined percentiles. Then $\bar{\gamma}$ is defined as

$\bar{\gamma}=\frac{1}{m} \sum_{i=1}^{m}\left|\gamma_{i}\right|$

where $\left|\gamma_{i}\right|$ is the absolute value of $\gamma_{i}$.

For example, in this study we calculate rank differences of each member between future projection and historical periods at the 5th, 10th, 20th, 30th, 40th, 50th, 60th, 70th, 80th, 90th, and 95th percentiles. Therefore, in this study, $m$ $=11, \gamma_{1}$ corresponds to the median of rank difference at the 5th percentile, $\gamma_{2}$ corresponds to the median of rank difference at the 10th percentile, etc. Using the above formula, the rank constancy indicators of gmsta from the RCP85-clim MME (Fig. 2b) and the RCP85-individual MME (Fig. 2c) are $\bar{\gamma}=1.18$ and $\bar{\gamma}=13.09$, respectively. Hence, the rank distribution of the RCP85-clim MME is more consistent with the constancy of rank distribution condition than that of the RCP85-individual MME.

\subsection{Construct the Rank Distribution of the Observations in Historical Period}

To construct the rank distribution of the observations within a given MME in historical period, we need to combine the observations with the given MME to form an augmented matrix $\widetilde{A}$. Using the same example as that of section $3.1, \widetilde{\mathrm{A}}$ is a $82 \times 600$ matrix. By sorting each column of $\widetilde{\mathrm{A}}$ in ascending order and identifying the corresponding column position of the observations, we yield a time series consisting 600 ranks of the observations. Then, the rank histogram of the observations in historical period can be constructed from this time series.

\subsection{Adjust Rank Distribution to Constrain Future Projections}

In this study, we modify the rank histogram calibration method (Hamill and Colucci 1997, 1998) to adjust the 'best-guess' and the 5 - 95 uncertainty ranges of future projections for a given variable from the corresponding MME. Conceptually, by identifying the ranks of the 5th (P5), 50th (P50), and 95th (P95) percentiles from the rank histogram of the observations, we can use these ranks to calibrate the median and the 5 - 95 uncertainty range of the observations derived from the corresponding MME in any period. However, because there is no observation in future projection period, the total rank of the MME in future projection period is reduced by 1 compared to that in historical period. Therefore, we need to adjust these ranks before applying them to 
constrain the estimation of the 'best-guess' and the 5 - 95 uncertainty range from the given MME.

It is noted that $\mathbf{P 5}$ implies there is 5\% (95\%) probability of occurrence that the rank of observations will be smaller (larger) than P5. If one removes the observations from the MME, then there is 5\% (95\%) probability that the rank of the 5 th percentile will be reduced by 1 (i.e., the rank is reduced to $\mathbf{P} 5-1$ ), while there is $95 \%$ probability that the rank of the 5th percentile will remain the same (i.e., the rank remains the same as $\mathbf{P 5}$ ). Therefore, when the observations are absent, the rank of the 5th percentile should be adjusted to $\mathbf{P} \tilde{5}=0.05 \times(\mathbf{P} 5-1)+0.95 \times \mathbf{P} 5$. However, because the median of rank difference at the 5th percentile $\left(\gamma_{1}\right)$ can be viewed as an optimal trend estimation of rank at this percentile, $\gamma_{1}$ should be added to the adjusted ranks in future projection period to account for the presence of this nonparametric trend. Therefore, the adjusted rank of the 5th (denoted as $\mathbf{P 5}^{\prime}$ ) percentile of the observations in future projection period can be estimated as,

$\mathbf{P} 5^{\prime}=\mathbf{P} \tilde{5}+\gamma_{1}=0.05 \times(\mathbf{P} 5-1)+0.95 \times \mathbf{P} 5+\gamma_{1}$

Similarly, the adjusted ranks of the 50th (P50') and 95th ( $\left.\mathbf{P} 95^{\prime}\right)$ percentiles of the observations in future projection period can be estimated as

$$
\mathbf{P} 50^{\prime}=\mathbf{P} \widetilde{50}+\gamma_{6}=0.5 \times(\mathbf{P} 50-1)+0.5 \times \mathbf{P} 50+\gamma_{6}
$$

and

$$
\mathbf{P} 95^{\prime}=\mathbf{P} \widetilde{95}+\gamma_{11}=0.95 \times(\mathbf{P} 95-1)+0.05 \times \mathbf{P} 95+\gamma_{11}
$$

where $\gamma_{6}$, and $\gamma_{11}$ are the medians of rank differences at the 50th and 95th percentiles, respectively. Then, we can use these adjusted ranks to constrain the median and the 5 95 uncertainty range of the corresponding variable in future projection period from the given MME.

\section{RESULTS}

Figure 3 shows the original and constrained medians and 5 - 95 uncertainty ranges of the gmsta from the RCP85clim MME and the RCP85-individual MME, respectively. In Fig. 3a, one notes that the original 5 - 95 uncertainty ranges of the RCP85-clim MME are over-dispersive but remain approximately constant during the whole period. Furthermore, the medians of the MME exhibit slightly cold bias in historical period compared to the observations. In contrast, the constrained 5 - 95 uncertainty ranges shown in Fig. 3c are very compact and narrow. Compared to Fig. 3a, these 5 - 95 uncertainty ranges around the end of the 21 st century have been successfully narrowed from $3^{\circ} \mathrm{K}$ $-6^{\circ} \mathrm{K}$ to $4^{\circ} \mathrm{K}-5.5^{\circ} \mathrm{K}$. Furthermore, the constrained medians show no visible bias with the observations in historical period. These results demonstrate that the adjustment procedure can effectively reduce biases and uncertainty ranges of gmsta's future projections from the RCP85-clim MME. On the other hand, Figs. $3 \mathrm{~b}$ and d show quite different characteristics. The medians in both panels clearly overlaps with the observations in historical period and the 5 - 95 uncertainty ranges tend to center around the observations in period 1961 - 1990 but gradually disperse toward both ends. Furthermore, one notes in Fig. 3d that the constrained 5 - 95 uncertainty ranges are too narrow to cause a sizable fraction of observations after year 2000 being left outside of the range. Because the rank distribution of RCP85-individual MME is not consistent with the constancy of rank distribution condition, this result suggests that a naïve application of the adjustment procedure to an already mean-biasremoved MME may lead to over-constrained consequence. Thus, it appears that if the rank distribution of a given MME is consistent with the constancy of rank distribution condition, then the rank adjustment procedure can properly constrain biases and uncertainty ranges of the corresponding variable's future projections.

Can this rank adjustment procedure be applied to individual grids and to other variables to constrain the corresponding future projections? To examine these possibilities, we first calculated the rank constancy indicator $(\bar{\gamma})$ of surface temperature and precipitation fields from both the RCP85-clim MME and the RCP85-individual MME at each grid on the global. Figure 4 shows the corresponding results. For surface temperature fields, Fig. 4a shows most grids except those around northern polar region have $\bar{\gamma}<2$, while most grids in Fig. $4 \mathrm{c}$ have $\bar{\gamma}>2$. As noted in Fig. 2, these results indicate that the rank distributions of surface temperature anomalies at most places from the RCP85-clim MME are more consistent with the constancy of rank distribution condition than those from the RCP85-individual MME. However, anomalies around northern polar region from both MMEs are inconsistent with the constancy of rank distribution condition. Furthermore, differences in $\bar{\gamma}$ between these two MMEs are larger in ocean areas than those in land areas. Because the main difference between these two MMEs is whether the mean bias in each model was removed, this result suggests that surface temperature warming trends in land areas may be more consistent among members of the CMIP5 MME than those in ocean areas.

As for precipitation fields, most grids in both Figs. $4 \mathrm{~b}$ and $\mathrm{d}$ have $\bar{\gamma}<1$. Grids with $\bar{\gamma}>1$ mainly locate within the Tropics. Nevertheless, no $\bar{\gamma}$ value is larger than 3 . Furthermore, although generally $\bar{\gamma}$ values from the RCP85clim MME are still less than those from the RCP85-individual MME at most places, the differences between them are small. These results indicate that the rank distributions of precipitation anomalies at most places from both MMEs are 

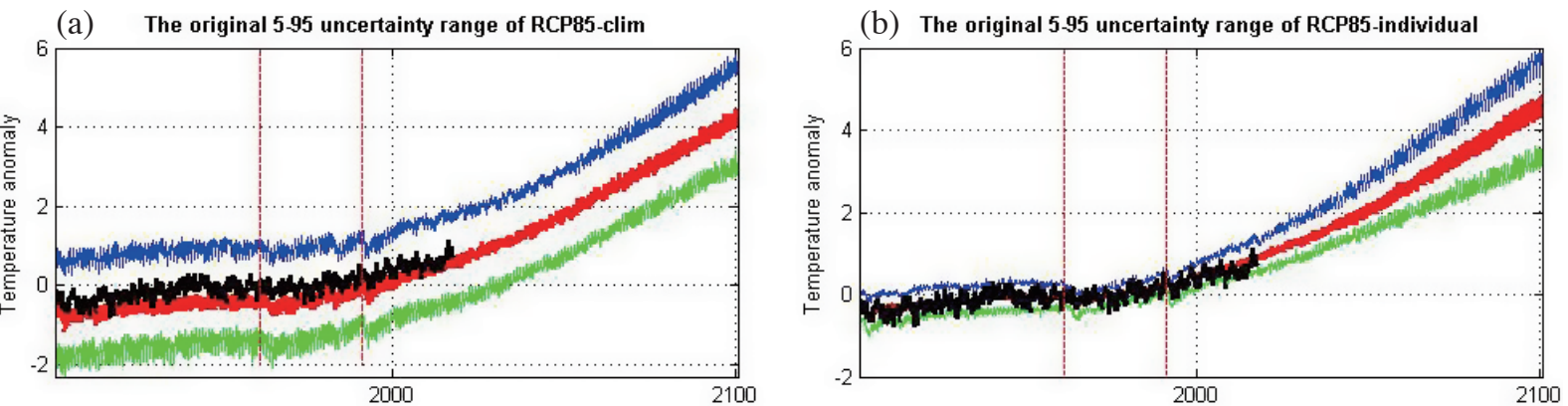

(c) The constrained 5-95 uncertainty range of RCP85-clim
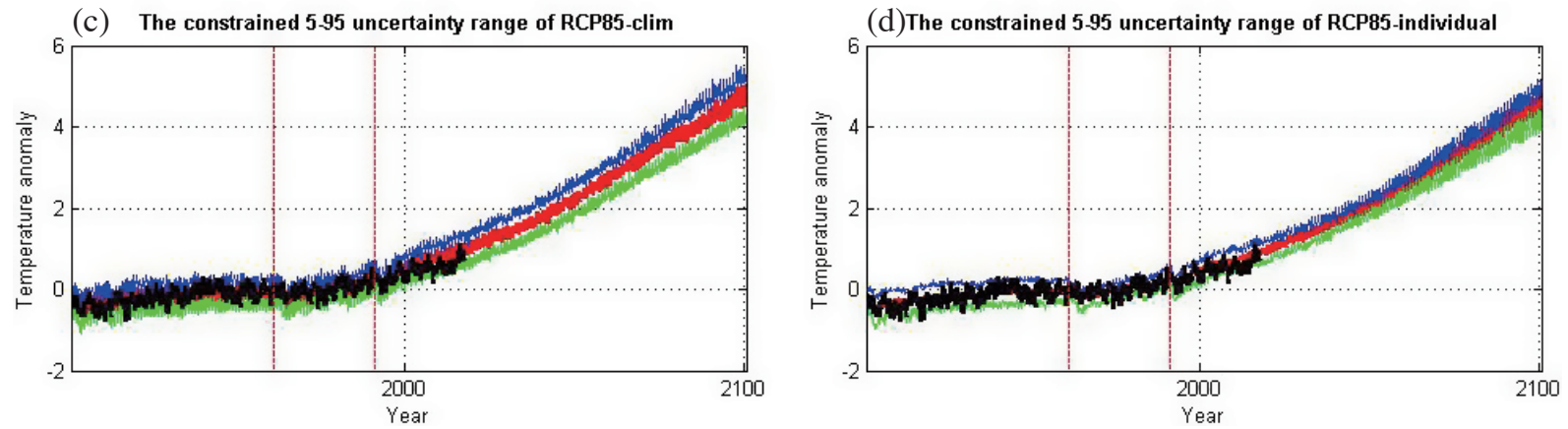

Fig. 3. The original and constrained median and 5 - 95 uncertainty range of the gmsta from the RCP85-clim MME (a) (c) and the RCP85-individual MME (b) (d), respectively. Blue, red, and green lines are lines of the 95th, 50th, and 5th percentiles, respectively. These lines are determined as follows. In period $1901-2000, \mathbf{P} \tilde{5}, \mathbf{P} \widetilde{50}$, and $\mathbf{P} \widetilde{95}$ are used. In period $2051-2100, \mathbf{P} 5^{\prime}, \mathbf{P} 50^{\prime}$, and P95' are used. In period $2001-2050$, the corresponding ranks are increased linear from $\mathbf{P} \tilde{5}, \mathbf{P} \widetilde{50}$, and $\mathbf{P} \widetilde{95}$ to $\mathbf{P} 5^{\prime}, \mathbf{P} 50^{\prime}$, and $\mathbf{P} 95^{\prime}$, respectively. Black line in each panel corresponds to the observations. The red vertical lines in each panel mark the range of reference period (years 1961 and 1990, respectively).

(a) Rank constancy indicator for RCP85-clim tas

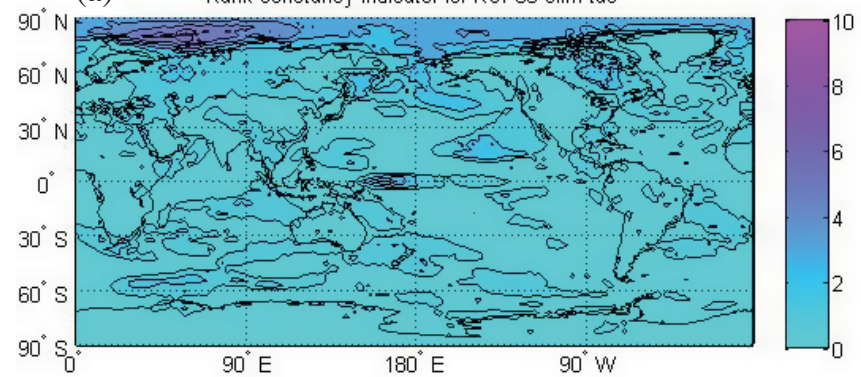

(c)
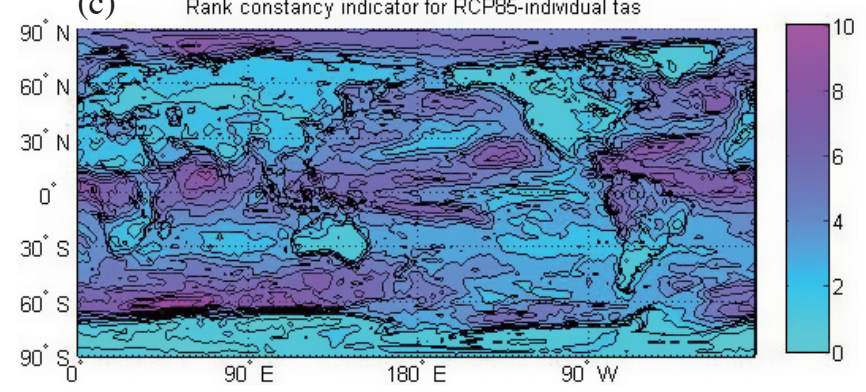

(b)

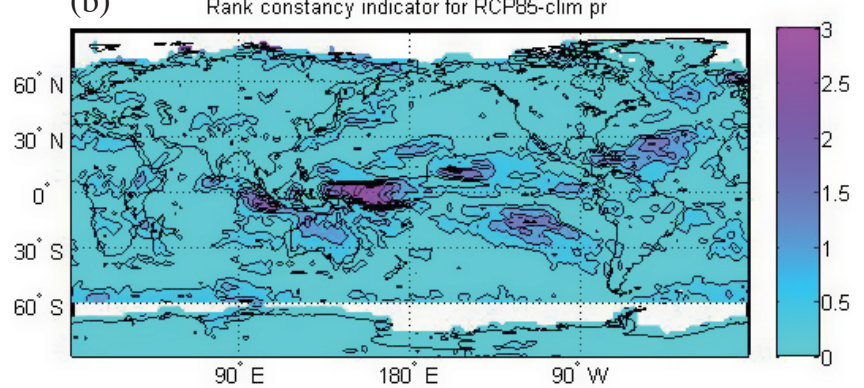

(d)

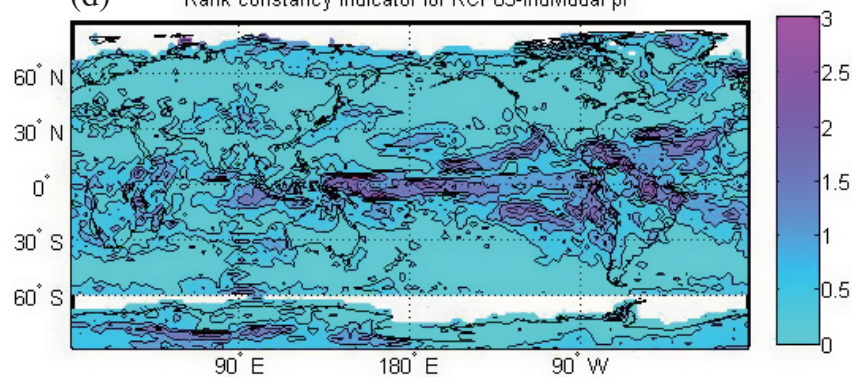

Fig. 4. Spatial distributions of the rank constancy indicator $(\bar{\gamma})$ of surface temperature (a) (c) and precipitation fields (b) (d) from the RCP85-clim MME and the RCP85-individual MME, respectively. 
consistent with the constancy of rank distribution condition. The only notable exception is the equatorial central Pacific where $\bar{\gamma}$ values from the RCP85-clim MME are larger than those from the RCP85-individual MME. This result indicates that precipitation trends in this region exhibit less consistent behavior among members of the CMIP5 MME than those in elsewhere.

We then applied the rank adjustment procedure to examine whether future projections of surface temperature and precipitation fields can be properly constrained. Figure 5 shows the 2081 - 2100 averaged spatial distributions of the original and constrained medians of both surface temperature and precipitation fields from the RCP85-clim MME. One notes in Fig. 5a that the original RCP85-clim MME medians of surface temperature anomalies exhibit significant spatial variabilities. Furthermore, although most places exhibit warming, still slightly cooling exist in some places (e.g., mid northern Atlantic Ocean between 45 and $60^{\circ} \mathrm{N}$ and Antarctic continent near dateline). In particular, anomalies around Antarctic are very erratic and some change signs over very short distances. In contrast, constrained medians in Fig. 5c are all positive and exhibit smoother and more homogeneous spatial variations than those in Fig. 5a. These medians generally follow land-sea distribution and tend to increase in magnitudes from low latitudes toward high latitudes. Furthermore, erratic variations around Antarctic are absent. These characteristics of Fig. $5 \mathrm{c}$ are quite similar to those of the 1988 - 2017 mean field of the HadCRUT4.6 shown in Fig. 6a. Because the HadCRUT4.6 data are surface temperature anomalies deviated from the 1961 - 1990 mean seasonal cycle, spatial pattern of Fig. 6a is equivalent to the mean climate change pattern of the 1988 - 2017 period. Thus, the resemblance between Figs. $5 \mathrm{c}$ and $6 \mathrm{a}$ suggests that the rank adjustment procedure is capable of properly constraining the corresponding future projections by selecting a subset of ranks that are consistent with those of observations from the given MME.

As for the precipitation field, constrained results are even more encouraging. In Fig. 5b one notes that the original RCP85-clim MME medians of precipitation anomalies exhibit a clear double intertropical convergence zone (ITCZ) pattern (Mechoso et al. 1995; Lin 2007) in the tropical Pacific. Because the double-ITCZ pattern is a common precipitation bias in many climate models, its appearance in Fig. 5b is not unexpected but is likely spurious. In contrast, the constrained medians of the precipitation anomalies shown in Fig. 5d exhibits a more realistic spatial pattern that resembles the 1988 - 2017 mean field of the CMAP shown in Fig. 6b in the equatorial Tropics. More interestingly, there is no spurious ITCZ pattern in the tropical south Pacific. Since this resemblance is very pronounced in the ITCZ region, the constrained future projection of precipitation field shown in Fig. 5d appears to be consistent with the rich-get-richer mechanism of precipitation change under global warming (Chou and Neelin 2004; Held and Soden 2006; Chou et al. 2009). Again, this result suggests that the rank adjustment procedure is capable of properly constraining the corresponding future projections by selecting a subset of ranks that are consistent with those of observations from the given MME.

Figure 7 shows the same as Fig. 5, except for results from the RCP85-individual MME. Look at this figure, one immediately notes the resemblance of spatial patterns between original and constrained results in both fields. Furthermore, the spatial patterns of surface temperature anomalies are similar to that of Fig. 6a. However, both spatial patterns of precipitation exhibit a clear double-ITCZ pattern. Because the mean bias of each model had been removed in constructing the RCP85-individual MME, both fields show no obvious differences between original and constrained results. Thus it appears that the rank adjustment procedure does not have any visible capability to better constrain the corresponding future projections from the RCP85individual MME.

Figures 8 and 9 show the $2081-2100$ averaged spatial distributions of the constrained 5 - 95 uncertainty ranges for both surface temperature and precipitation fields as well as the corresponding differences between the constrained and the original 5 - 95 uncertainty ranges from the RCP85-clim MME and the RCP85-individual MME, respectively. In Fig. 8a one clearly notes that the calibrated uncertainty ranges have distinct land-sea contrast with magnitudes over lands being significantly larger than those over oceans. Furthermore, both Arctic and Antarctic regions have significantly greater uncertainty ranges than elsewhere. These features are similar to those of the 1988 - 2017 standard deviation field of the HadCRUT4.6 shown in Fig. 6c. It is noted that spatial patterns of both the uncertainty range and the standard deviation fields characterize the spatial distribution of a given variable's temporal variability. Furthermore, temporal variability of a given variable at individual grid is an integral response of the dynamic and thermodynamic properties of that specific place. Therefore, if global climate change does not introduce substantial change in local dynamic and thermodynamic properties in most places on the earth, this resemblance of spatial patterns between the uncertainty range and the standard deviation fields may be unexpected. Similar features are also observed in Fig. 9a. Nevertheless, the constrained uncertainty ranges in Fig. 8a are generally smaller than those in Fig. 9a. An obvious exception is those in the Antarctic region where the constrained uncertainty ranges in Fig. 8a are larger than those in Fig. 9a. The corresponding spatial distributions of differences between the constrained and the original 5 - 95 uncertainty ranges shown in both Figs. 8c and 9c reveal that most of these differences are negative. Similarly, an obvious exception is those in the Antarctic region where the differences are positive. Furthermore, the magnitudes of these differences (regardless their 
(a) Median of original RCP85-clim tas between 2081-2100

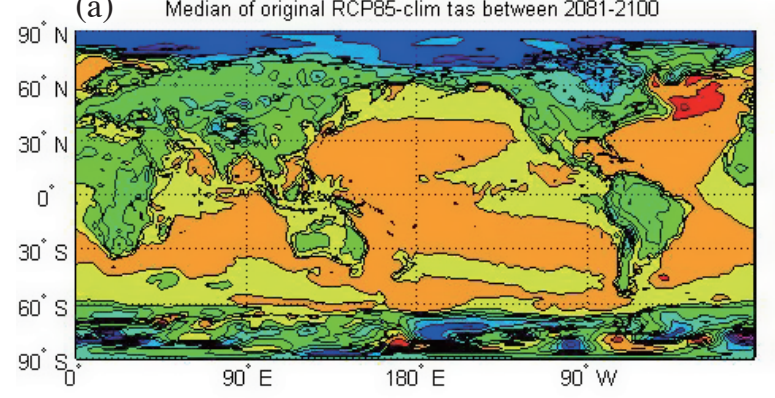

(c) Median of constrained RCP85-clim tas between 2081-2100
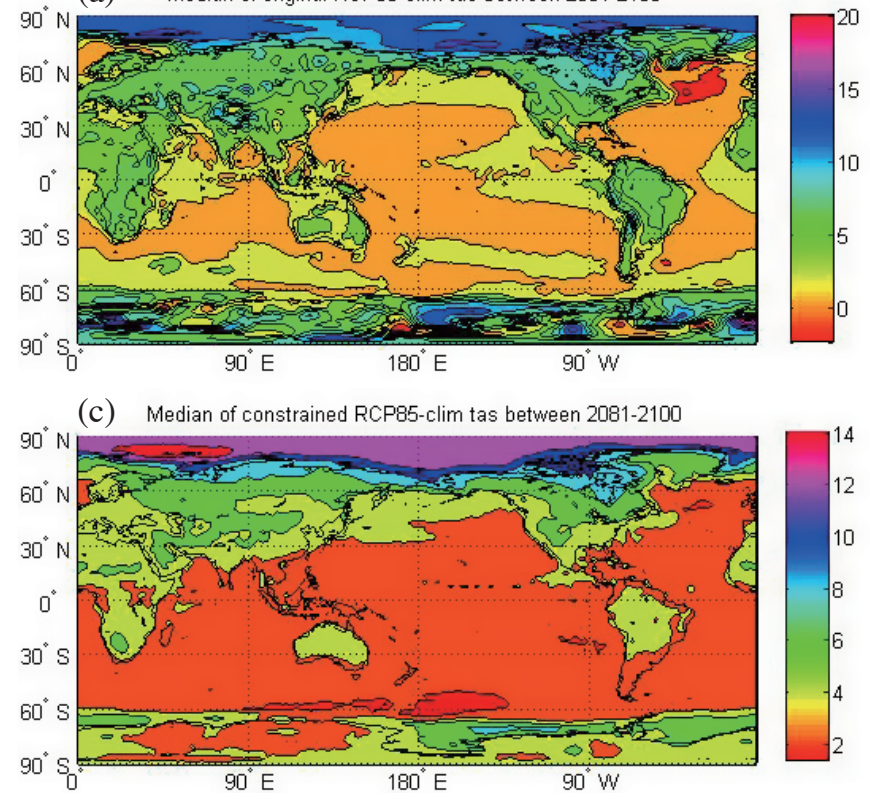

(b) Median of original RCP85-clim pr between 2081-2100

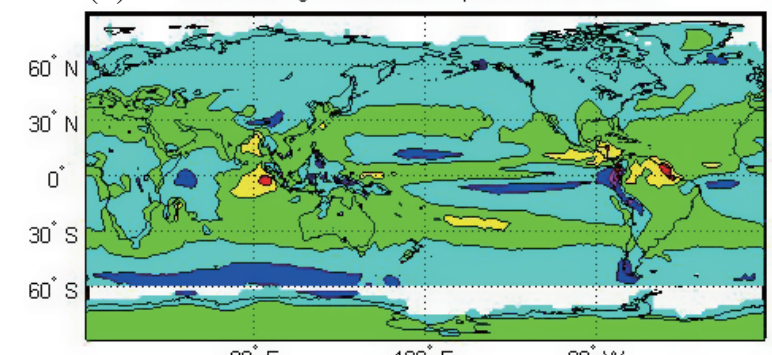

$90^{\circ} \mathrm{E}$

$180^{\circ} \mathrm{E}$

$90^{\circ} \mathrm{W}$

(d) Median of constrained RCP85-clim pr between 2081-2100

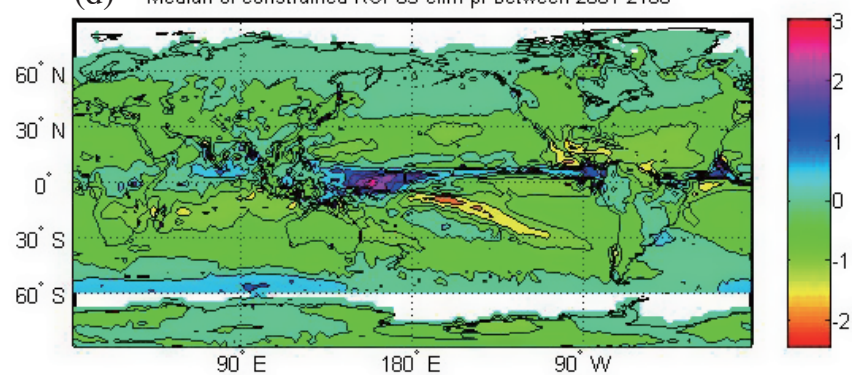

Fig. 5. The 2081 - 2100 averaged spatial distributions of the original (a) and the constrained (c) surface temperature projections from the RCP85clim MME medians. (b) and (d) are the corresponding spatial patterns for precipitation projections.

(a)

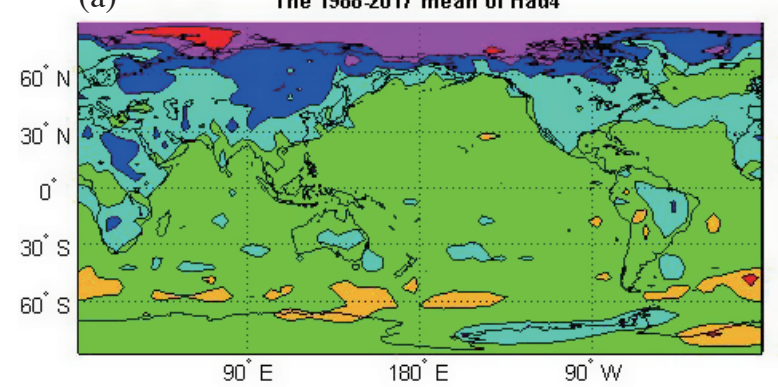

(c)

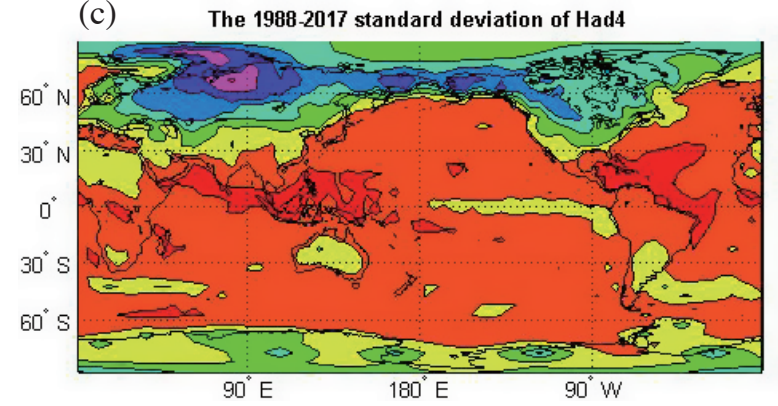

(b)
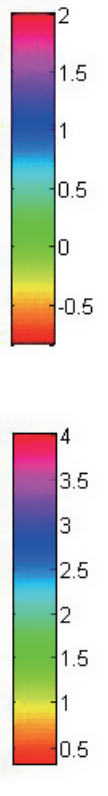
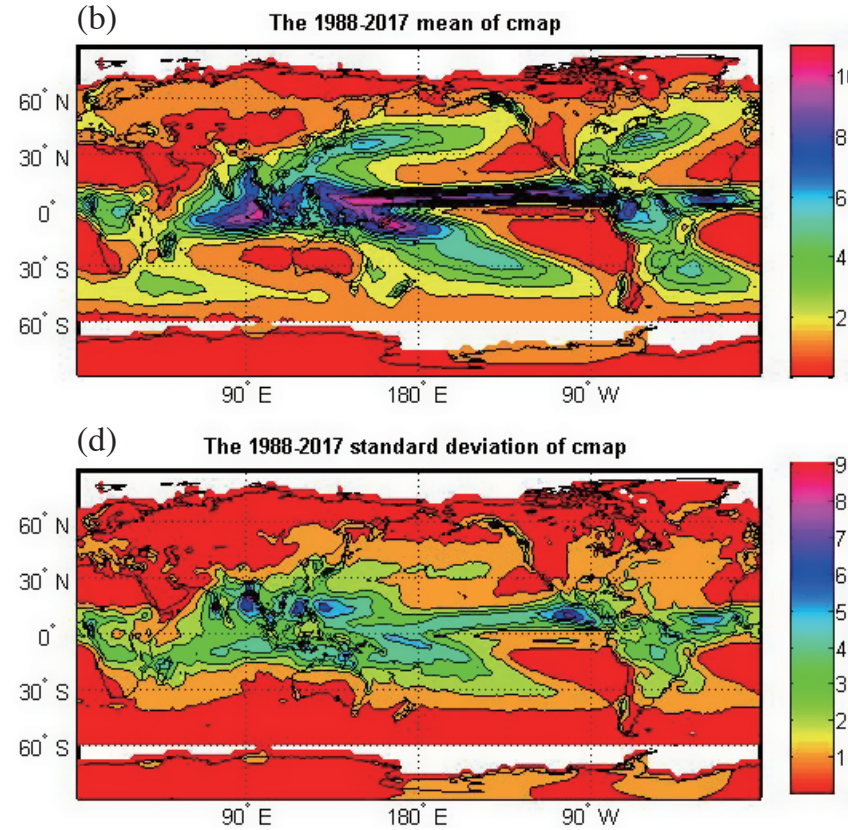

Fig. 6. The 1988 - 2017 mean and standard deviation fields of the HadCRUT4.6 surface temperature anomalies and the CMAP precipitation, respectively. 
(a) Median of original RCP85-individual tas between 2081-2100

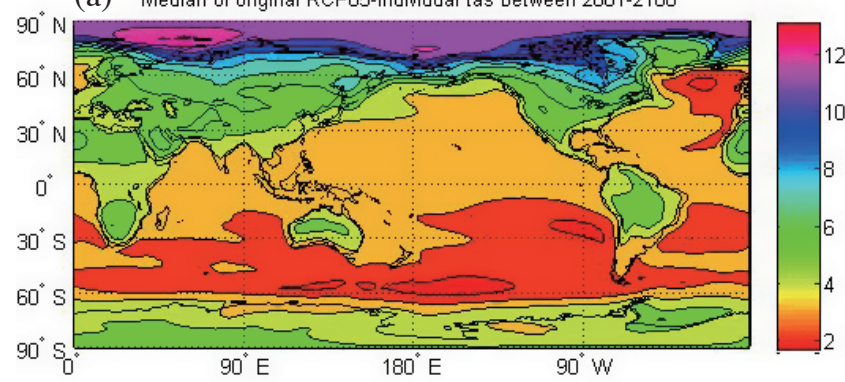

(c) Median of constrained RCP85-individual tas between 2081-2100

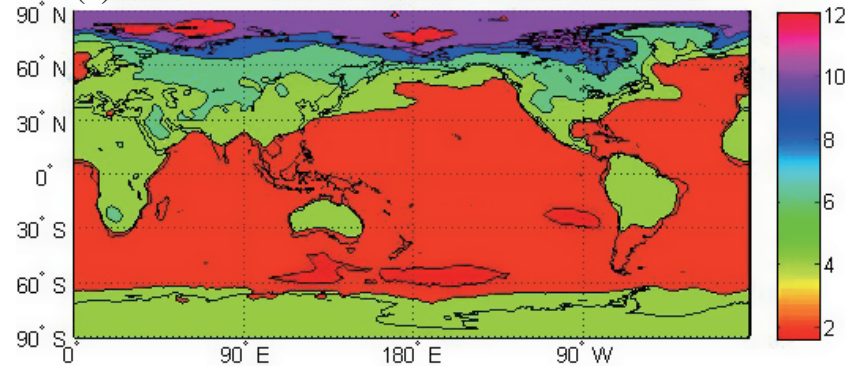

(b) Median of original RCP85-individual pr between 2081-2100

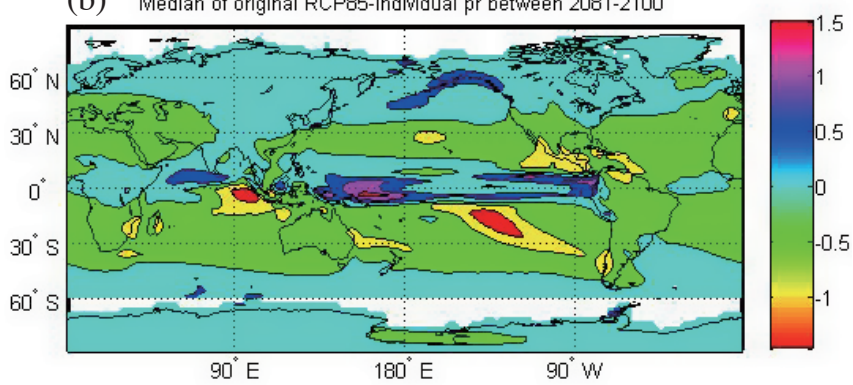

(d) Median of constrained RCP85-individual pr between 2081-2100

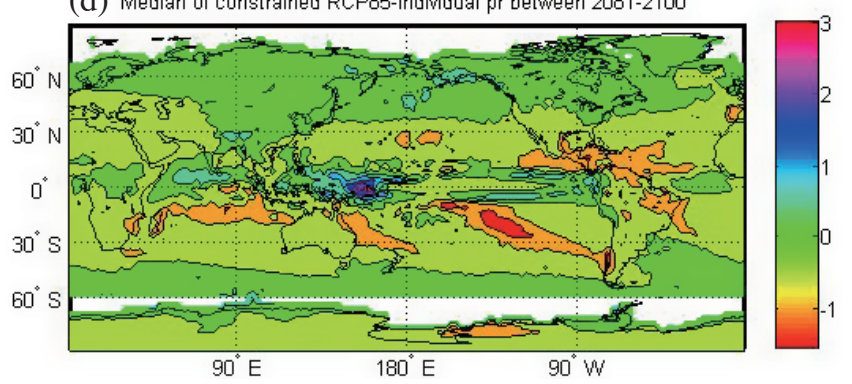

Fig. 7. The same as Fig. 5, except for results from the RCP85-individual MME.

(a) Constrained 5-95 range of RCP85-clim tas between 2081-2100

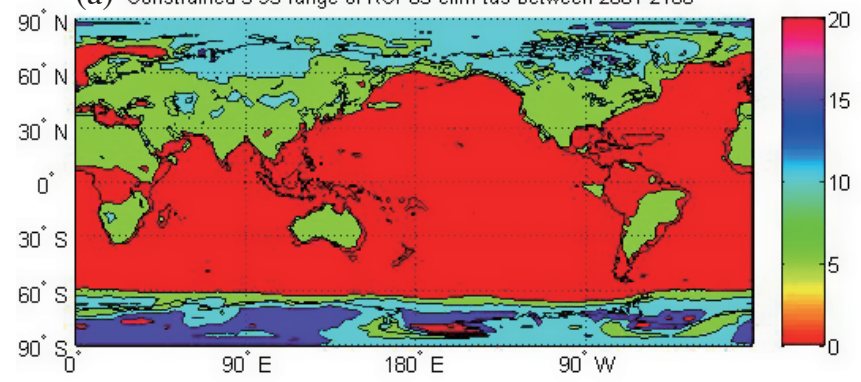

(c) Difference in 5-95 range between constrained and original tas

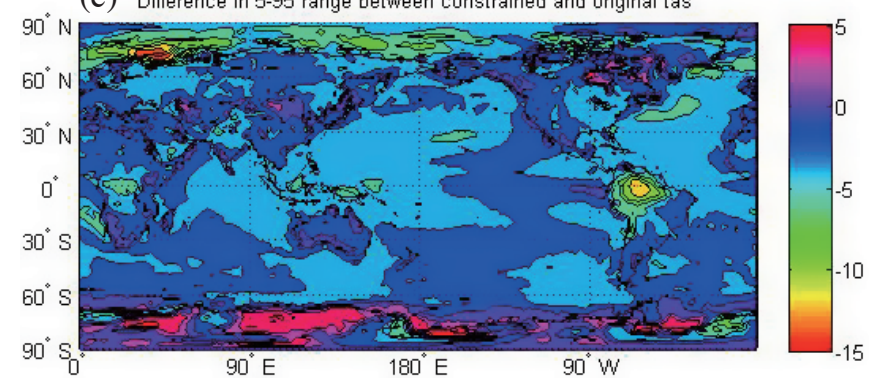

(b) Constrained 5-95 range of RCP85-clim pr between 2081-2100

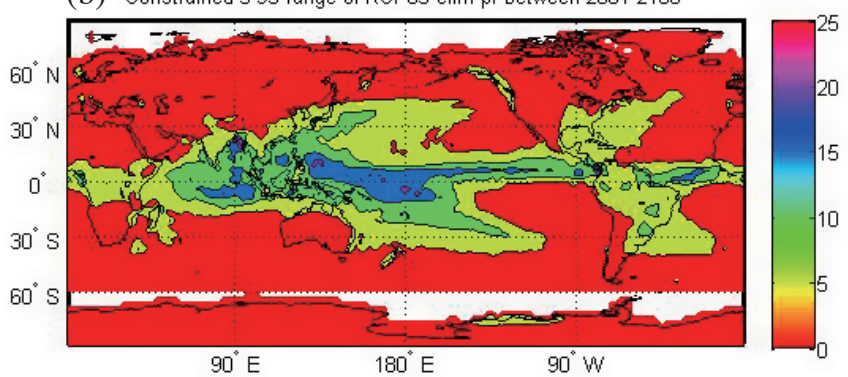

(d) Difference in 5-95 range between constrained and original pr

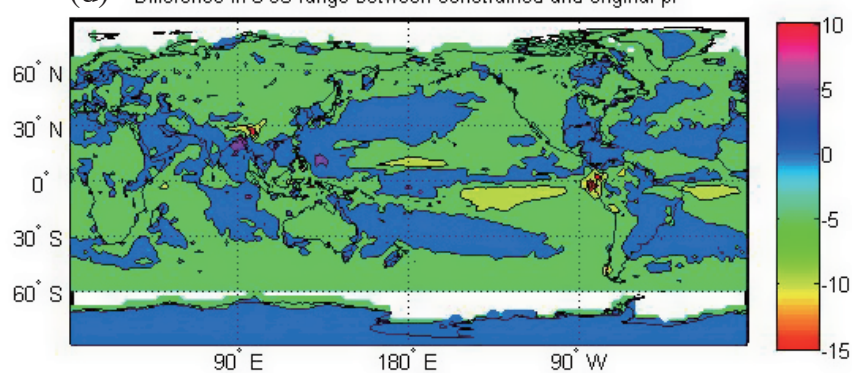

Fig. 8. The 2081 - 2100 averaged spatial distributions of the constrained 5 - 95 uncertainty ranges for surface temperature (a) and precipitation (b) fields from the RCP85-clim MME. The corresponding spatial distributions of differences between the constrained and the original 5 - 95 uncertainty ranges are shown in (c) and (d). Negative values in both panels indicate that the constrained 5 - 95 uncertainty ranges are less than original ranges in those places. 
(a) Constrained 5-95 range of RCP85-individual tas between 2081-2100

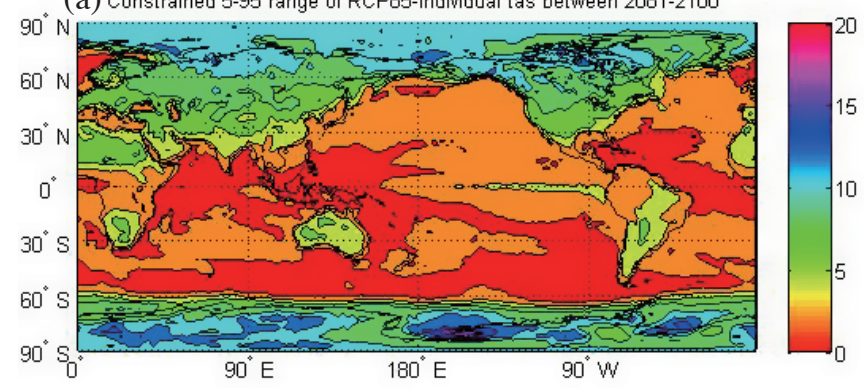

(c) Difference in 5-95 range between constrained and original tas

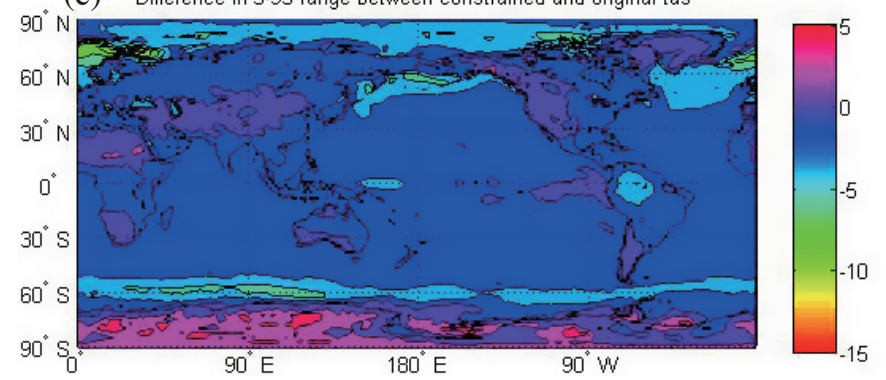

(b) Constrained 5-95 range of RCP85-individual pr between 2081-2100

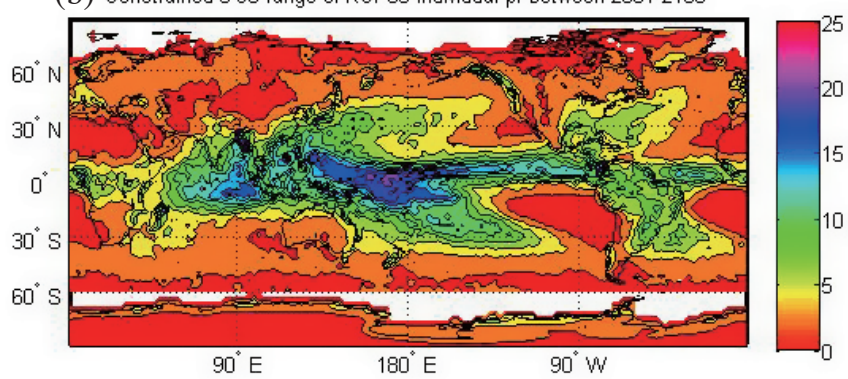

(d) Difference in 5-95 range between constrained and original pr

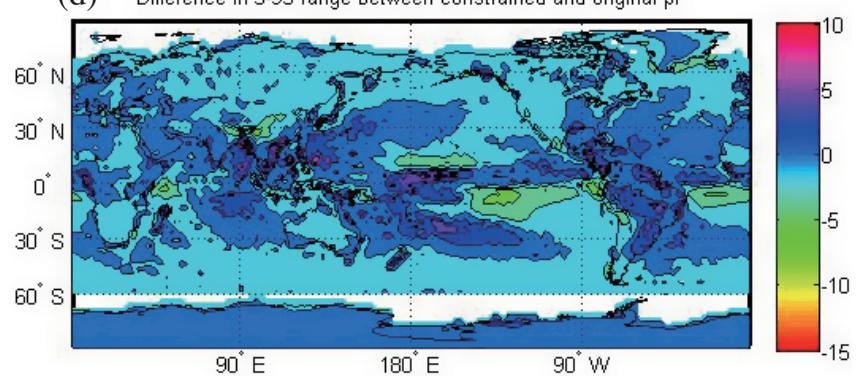

Fig. 9. The same as Fig. 8, except for results from the RCP85-individual MME.

signs) in Fig. 8c are generally larger than those in Fig. 9c. These results demonstrate that the rank adjustment procedure can indeed reduce uncertainty ranges of surface temperature projections at most places. Furthermore, the rank adjustment procedure is more effective in constraining future projections from the RCP85-clim MME than from the RCP85-individual MME. However, the results also show there are places where the rank adjustment procedure is unable to reduce the uncertainty range. Although the exact causes for this failure are yet to be explored, the spread of the given MME being under-dispersive or severely biased may be one of the possible reasons. In this respect, regions where this rank adjustment procedure failed may also provide valuable clues for model improvements.

As for the precipitation field, one notes that the spatial distribution of the constrained 5 - 95 uncertainty ranges in both Figs. $8 \mathrm{~b}$ and $9 \mathrm{~b}$ are quite similar to the $1988-2017$ standard deviation field of the CMAP (shown in Fig. 6d). The constrained uncertainty ranges in Fig. $8 \mathrm{~b}$ are also generally smaller than those in Fig. 9b. The corresponding spatial distributions of differences between the constrained and the original $5-95$ uncertainty ranges shown in both Figs. $8 \mathrm{~d}$ and $9 \mathrm{~d}$ reveal that most of these differences are negative. Furthermore, the magnitudes of these negative differences in Fig. 8d are generally larger than those in Fig. 9d. These results further confirm that the rank adjustment procedure can indeed be used to constrain future projections of a given MME at most places on the earth.

\section{DISCUSSION AND CONCLUSION}

In this study, we introduce a three steps rank adjustment procedure to explore the feasibility of using the rank distributions of a given MME to establish nonparametric presentfuture relations to be used in constraining the corresponding future projections. The first step is applying the perfect model approach to examine rank differences between future projection and historical periods as a function of percentiles from a given MME. It is noted that these rank differences can be viewed as the corresponding nonparametric trends of each member at these percentiles. This implies that the medians of rank differences of all members of a given MME can be viewed as an optimal estimation of the nonparametric trends of the given MME at these percentiles. Therefore, median of rank differences from each examined percentile is the desired nonparametric present-future relation we want to establish. The second step is to construct the rank distribution of the observations in the same historical period to be used to constrain the corresponding future projections. The third step is to adjust the rank distribution of the observations in the future projection period using the established present-future relationships to constrain the 'best-guess' and uncertainty ranges of future projections from the corresponding MME.

Afterward, we applied the rank adjustment procedure to gmsta from both RCP85-clim and RCP85-individual MMEs. The rank adjustment constrained results from the 
RCP85-clim gmsta show that the adjustment indeed has the capability to reduce the biases and uncertainty ranges of gmsta's future projections, while those from the RCP85-individual indicate that the adjustment tends to yield over-calibrated consequence. Then we applied the rank adjustment procedure to constrain future projections of surface temperature and precipitation fields. For surface temperature and precipitation fields, spatial patterns of the constrained medians averaged over 2081 - 2100 from the RCP85-clim MME are quite similar to that of the 1988 - 2017 mean field of the HadCRUT4.6 and the CMAP, respectively. These resemblances suggest that the rank adjustment procedure is capable of properly constraining the corresponding future projections by selecting a subset of ranks that are consistent with those of observations from the RCP85-clim MME. More interestingly, this resemblance in precipitation field implies that the constrained medians of precipitation projections are completely free from the long-standing double ITCZ bias. Furthermore, differences between the constrained and the original 5 - 95 uncertainty ranges of surface temperature and precipitation fields are mostly negative. These results confirm that the application of rank adjustment procedure can indeed reduce biases and uncertainty range of the corresponding future projections at most locations on the earth.

As for results from the RCP85-individual MME, the corresponding spatial patterns of the original and the constrained future projection results for both surface temperature and precipitation fields are quite similar. In particular, although being rank adjusted, the constrained medians of precipitation projections are still contaminated by the double ITCZ bias. These results indicate that the rank adjustment procedure has only insignificant impact on future projections derived from the RCP85-individual MME. Compare to results from the RCP85-clim MME, it appears that the combination of the rank adjustment procedure and the RCP85 MME generally yields more consistent future projections than other combinations.

We have demonstrated the feasibility of using the rank adjustment procedure to constrain the corresponding future projections from a given MME. Although results are encouraging, more studies and comparison with other related works are needed to improve the methodology, implementation, and applicability of the rank adjustment procedure. Nevertheless, because this procedure is based on nonparametric presentfuture relationships of a given MME, its ranges of applicability are believed to be wider than previously identified and proposed emergent constraints. As global warming is accelerating, we believe that this rank adjustment procedure can play an important role in providing more consistent future projections of surface temperature, precipitation fields and other related variables from the forthcoming CMIP6 (Eyring et al. 2016) MME to convince policy maker and public to take stronger actions to prevent foreseeable catastrophes.
Acknowledgements This study was supported by the Ministry of Science and Technology of Taiwan through grants MOST105-2111-M-008-004-, MOST1062111-M-008-014-, MOST107-2111-M-008-010-. The CMIP5 data used in this study can be accessed at http://pcmdi9.1lnl.gov// or http://climexp.knmi.nl/start.cgi, the HadCRUT4.6 data can be accessed at https://www.metoffice. gov.uk/hadobs/hadcrut4/, and the CMAP data can be accessed at https://www.esrl.noaa.gov/psd/data/gridded/data. cmap.html.

\section{REFERENCES}

Abe, M., H. Shiogama, J. C. Hargreaves, J. D. Annan, T. Nozawa, and S. Emori, 2009: Correlation between Inter-Model Similarities in Spatial Pattern for Present and Projected Future Mean Climate. SOLA, 5, 133136, doi: 10.2151/sola.2009-034. [Link]

Abramowitz, G. and C. H. Bishop, 2015: Climate Model Dependence and the Ensemble Dependence Transformation of CMIP Projections. J. Clim., 28, 2332-2348, doi: 10.1175/jcli-d-14-00364.1. [Link]

Anderson, J. L., 1996: A Method for Producing and Evaluating Probabilistic Forecasts from Ensemble Model Integrations. J. Clim., 9, 1518-1530, doi: 10.1175/1520-0442(1996)009<1518:Amfpae>2.0 . Co;2. [Link]

Borodina, A., E. M. Fischer, and R. Knutti, 2017: Emergent Constraints in Climate Projections: A Case Study of Changes in High-Latitude Temperature Variability. $J$. Clim., 30, 3655-3670, doi: 10.1175/jcli-d-16-0662.1. [Link]

Bracegirdle, T. J. and D. B. Stephenson, 2012: Higher precision estimates of regional polar warming by ensemble regression of climate model projections. Clim.Dyn.,39, 2805-2821, doi: 10.1007/s00382-012-1330-3. [Link]

Caldwell, P. M., C. S. Bretherton, M. D. Zelinka, S. A. Klein, B. D. Santer, and B. M. Sanderson, 2014: Statistical significance of climate sensitivity predictors obtained by data mining. Geophys. Res. Lett., 41, 18031808, doi: 10.1002/2014gl059205. [Link]

Carleton, T. A. and S. M. Hsiang, 2016: Social and economic impacts of climate. Science, $\mathbf{3 5 3}$, aad9837, doi: 10.1126/science.aad9837. [Link]

Chou, C. and J. D. Neelin, 2004: Mechanisms of Global Warming Impacts on Regional Tropical Precipitation. J. Clim., 17, 2688-2701, doi: 10.1175/1520-0442(2004)017<2688:Mogwio>2.0. Co;2. [Link]

Chou, C., J. D. Neelin, C.-A. Chen, and J.-Y. Tu, 2009: Evaluating the "Rich-Get-Richer" Mechanism in Tropical Precipitation Change under Global Warming. J. Clim., 22, 1982-2005, doi: 10.1175/2008jcli2471.1. [Link] 
DelSole, T. and J. Shukla, 2009: Artificial Skill due to Predictor Screening. J. Clim., 22, 331-345, doi: 10.1175/2008jcli2414.1. [Link]

Eyring, V., S. Bony, G. A. Meehl, C. A. Senior, B. Stevens, R. J. Stouffer, and K. E. Taylor, 2016: Overview of the Coupled Model Intercomparison Project Phase 6 (CMIP6) experimental design and organization. Geosci. Model Dev., 9, 1937-1958, doi: 10.5194/gmd-91937-2016. [Link]

Hall, A. and X. Qu, 2006: Using the current seasonal cycle to constrain snow albedo feedback in future climate change. Geophys. Res. Lett., 33, doi: 10.1029/2005g1025127. [Link]

Hall, A., P. Cox, C. Huntingford, and S. Klein, 2019: Progressing emergent constraints on future climate change. Nat. Clim. Change, 9, 269-278, doi: 10.1038/ s41558-019-0436-6. [Link]

Hamill, T. M., 2001: Interpretation of Rank Histograms for Verifying Ensemble Forecasts.Mon. Weather Rev.,129, 550-560, doi: 10.1175/1520-0493(2001)129<0550:Ior hfv>2.0.Co;2. [Link]

Hamill, T. M. and S. J. Colucci, 1997: Verification of Eta-RSM Short-Range Ensemble Forecasts. Mon. Weather Rev., 125, 1312-1327, doi: 10.1175/1520-0493(1997)125<1312: Voersr>2 .0. Co;2. [Link]

Hamill, T. M. and S. J. Colucci, 1998: Evaluation of Eta-RSM Ensemble Probabilistic Precipitation Forecasts. Mon. Weather Rev., 126, 711-724, doi: 10.1175/1520-0493(1998)126<0711:Eoerep>2.0. Co;2. [Link]

Held, I. M. and B. J. Soden, 2006: Robust Responses of the Hydrological Cycle to Global Warming. J. Clim., 19, 5686-5699, doi: 10.1175/jcli3990.1. [Link]

Herger, N., G. Abramowitz, R. Knutti, O. Angélil, K. Lehmann, and B. M. Sanderson, 2018: Selecting a climate model subset to optimise key ensemble properties. Earth Syst. Dynam., 9, 135-151, doi: 10.5194/esd-9135-2018. [Link]

Klein, S. A. and A. Hall, 2015: Emergent Constraints for Cloud Feedbacks. Current Climate Change Reports, $\mathbf{1}$, 276-287, doi: 10.1007/s40641-015-0027-1. [Link]

Knutti, R., 2008: Should we believe model predictions of future climate change? Phil. Trans. Math. Phys. Eng. Sci., 366, 4647-4664, doi: 10.1098/rsta.2008.0169. [Link]

Knutti, R., 2010: The end of model democracy? Clim. Change, 102, 395-404, doi: 10.1007/s10584-0109800-2. [Link]

Knutti, R., R. Furrer, C. Tebaldi, J. Cermak, and G. A. Meehl, 2010: Challenges in Combining Projections from Multiple Climate Models. J. Clim., 23, 27392758, doi: 10.1175/2009jcli3361.1. [Link]

Knutti, R., D. Masson, and A. Gettelman, 2013: Climate model genealogy: Generation CMIP5 and how we got there. Geophys. Res. Lett., 40, 1194-1199, doi: 10.1002/grl.50256. [Link]

Knutti, R., J. Sedláček, B. M. Sanderson, R. Lorenz, E. M. Fischer, and V. Eyring, 2017: A climate model projection weighting scheme accounting for performance and interdependence. Geophys. Res. Lett., 44, 1909-1918, doi: 10.1002/2016g1072012. [Link]

Krishnamurti, T. N., C. M. Kishtawal, Z. Zhang, T. LaRow, D. Bachiochi, E. Williford, S. Gadgil, and S. Surendran, 2000: Multimodel Ensemble Forecasts for Weather and Seasonal Climate. J. Clim., 13, 41964216, doi: 10.1175/1520-0442(2000)013<4196:Meffw a 2.0. Co;2. [Link]

Li, G., S.-P. Xie, and Y. Du, 2016: A Robust but Spurious Pattern of Climate Change in Model Projections over the Tropical Indian Ocean. J. Clim., 29, 5589-5608, doi: 10.1175/jcli-d-15-0565.1. [Link]

Lin, J.-L., 2007: The Double-ITCZ Problem in IPCC AR4 Coupled GCMs: Ocean-Atmosphere Feedback Analysis. J. Clim., 20, 4497-4525, doi: 10.1175/jcli4272.1. [Link]

Masson, D. and R. Knutti, 2011: Climate model genealogy. Geophys. Res. Lett., 38, doi: 10.1029/2011g1046864. [Link]

Masson, D. and R. Knutti, 2013: Predictor Screening, Calibration, and Observational Constraints in Climate Model Ensembles: An Illustration Using Climate Sensitivity. J. Clim., 26, 887-898, doi: 10.1175/jclid-11-00540.1. [Link]

Mechoso, C. R., A. W. Robertson, N. Barth, M. K. Davey, P. Delecluse, P. R. Gent, S. Ineson, B. Kirtman, M. Latif, H. Le Treut T. Nagai, J. D. Neelin, S. G. H. Philander, J. Polcher, P. S. Schopf, T. Stockdale, M. J. Suarez, L. Terray, O. Thual, and J. J. Tribbia, 1995: The Seasonal Cycle over the Tropical Pacific in Coupled Ocean-Atmosphere General Circulation Models. Mon. Weather Rev., 123, 2825-2838, doi: 10.1175/1520-0493(1995)123<2825:Tscott>2.0.Co;2. [Link]

Mirzargar, M. and J. L. Anderson, 2017: On Evaluation of Ensemble Forecast Calibration Using the Concept of Data Depth. Mon. Weather Rev., 145, 1679-1690, doi: 10.1175/mwr-d-16-0351.1. [Link]

Morice, C. P., J. J. Kennedy, N. A. Rayner, and P. D. Jones, 2012: Quantifying uncertainties in global and regional temperature change using an ensemble of observational estimates: The HadCRUT4 data set. J. Geophys. Res., 117, doi: 10.1029/2011jd017187. [Link]

Robertson, A. W., U. Lall, S. E. Zebiak, and L. Goddard, 2004: Improved Combination of Multiple Atmospheric GCM Ensembles for Seasonal Prediction. Mon. Weather Rev., 132, 2732-2744, doi: 10.1175/ mwr2818.1. [Link] 
Sanderson, B. and R. Knutti, 2012: Climate Change Projections: Characterizing Uncertainty Using Climate Models. In: Meyers, R. A. (Ed.), Encyclopedia of Sustainability Science and Technology, Springer, New York, 2097-2114, doi: 10.1007/978-1-4419-0851-3_369. [Link]

Sanderson, B. M., R. Knutti, and P. Caldwell, 2015: Addressing Interdependency in a Multimodel Ensemble by Interpolation of Model Properties. J. Clim., 28, 5150-5170, doi: 10.1175/jcli-d-14-00361.1. [Link]

Taylor, K. E., R. J. Stouffer, and G. A. Meehl, 2012: An Overview of CMIP5 and the Experiment Design. Bull.
Am. Meteorol. Soc., 93, 485-498, doi: 10.1175/bamsd-11-00094.1. [Link]

Tebaldi, C. and R. Knutti, 2007: The use of the multi-model ensemble in probabilistic climate projections. Phil. Trans. Math. Phys. Eng. Sci., 365, 2053-2075, doi: 10.1098/rsta.2007.2076. [Link]

Xie, P. and P. A. Arkin, 1997: Global Precipitation: A 17Year Monthly Analysis Based on Gauge Observations, Satellite Estimates, and Numerical Model Outputs. Bull. Am. Meteorol. Soc., 78, 2539-2558, doi: 10.1175/1520-0477(1997)078<2539:Gpayma>2.0. Co;2. [Link] 